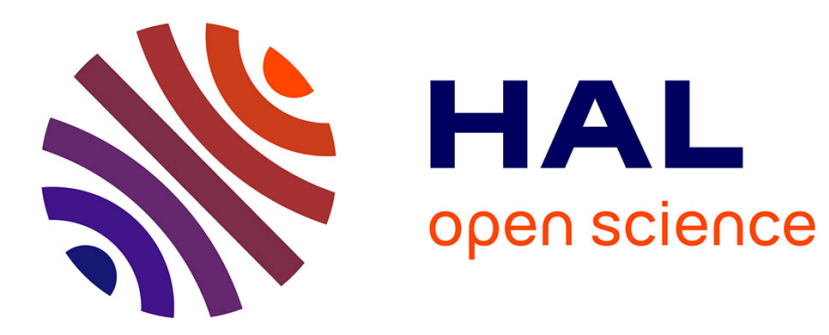

\title{
Geometrical representations in the learning of two-variable functions
}

Maria Trigueros, Rafael Martínez-Planell

\section{To cite this version:}

Maria Trigueros, Rafael Martínez-Planell. Geometrical representations in the learning of two-variable functions. Educational Studies in Mathematics, 2010, 73 (1), pp.3-19. 10.1007/s10649-009-9201-5 . hal-03409531

\section{HAL Id: hal-03409531 \\ https://hal.science/hal-03409531}

Submitted on 29 Oct 2021

HAL is a multi-disciplinary open access archive for the deposit and dissemination of scientific research documents, whether they are published or not. The documents may come from teaching and research institutions in France or abroad, or from public or private research centers.
L'archive ouverte pluridisciplinaire HAL, est destinée au dépôt et à la diffusion de documents scientifiques de niveau recherche, publiés ou non, émanant des établissements d'enseignement et de recherche français ou étrangers, des laboratoires publics ou privés. 
The following is the authors' copy.

The final publication is available at: http://ink.springer.com/article/10.1007\%2Fs10649-009-9201-5

/running head: TWO VARIABLE FUNCTIONS

title: GEOMETRICAL REPRESENTATIONS IN THE LEARNING OF TWO VARIABLE

\section{FUNCTIONS}

author(s):

Maria Trigueros Gaisman

trigue@itam.mx

Instituto Tecnológico Autónomo de México
Rafael Martínez-Planell

rafael@math.uprm.edu

University of Puerto Rico at Mayagüez

full address for correspondence:

María Trigueros

Depto. de Matemáticas

Instituto Tecnológico Autónomo de México

Río Hondo \#1

Tizapán, San Angel

México DF 01000

México

Phone number: (5255) $56284000 \times 3815$

Fax: (5255) 5536-1858

Email: trigue@itam.mx 
GEOMETRICAL REPRESENTATIONS IN THE LEARNING OF TWO VARIABLE FUNCTIONS

Maria Trigueros Gaisman

trigue@itam.mx

Instituto Tecnológico Autónomo de Mexico
Rafael Martínez-Planell

rafael@math.uprm.edu

University of Puerto Rico at Mayagüez

\begin{abstract}
This study is part of a project concerned with the analysis of how students work with two variable functions. This is of fundamental importance given the role of multivariable functions in mathematics and its applications. The portion of the project we report here concentrates on investigating the relationship between students' notion of subsets of Cartesian three dimensional space, and the understanding of graphs of two variable functions. APOS theory and Duval's theory of semiotic representations are used as theoretical framework. Nine students, who had taken a multivariable calculus course, were interviewed. Results show that students' understanding can be related to the structure of their schema for $\mathrm{R}^{3}$ and to their flexibility in the use of different representations.
\end{abstract}

Key words: APOS, graphic representation, schema, two variable functions, representations

\title{
1. Introduction
}


The notion of a multivariable function is fundamental in mathematics and its applications. Research on this subject, however, is scarce. There are many studies that deal with the general idea of a function; however, few make use of the particularities of multivariable functions to explicitly study how students build their understanding about them. While reviewing the literature, we could not find theory based research studies probing student understanding of this notion. This lack of research findings limits the understanding of how students learn the main ideas involved in the study of multivariable calculus. The purpose of this study is to contribute to filling this gap in the literature. In this paper, we focus on the analysis of some geometrical factors that are related to students' notion of function of two variables.

Understanding the geometrical properties of functions involves the possibility of relating different representations of functions. This in turn is related with visualization. During the last two decades, the critical problem of translation between and within representations has been addressed in several studies. For example, Breidenbach, Hawks, Nichols, and Dubinsky (1992) and Sfard (1992) propose that the ability of recognizing and being able to build a bridge between algebraic and graphic representations of functions differentiates between students who have and those who have not encapsulated the notion of function. Referring to the generalization involved in the transition from functions of one variable to functions of two variables, Yerushalmy (1997) insisted on the importance of the interplay between different representations to generalize key aspects of these functions and to identify changes in what seemed to be fixed properties of each type of function or representation. More recently, Gagatsis, Christou, and Elia (2004) argued that representations constitute different entities and that as such require explicit instruction. 
A competent use of different representations for a concept has been related to visualization. Gutierrez (1996), Goldin (1998, 2002), Hitt (2002) and, Van Nes and De Lange (2007), among other researchers, insist on the relationship between research on representations, mathematical visualization and understanding of concepts. Many research papers have studied students' visualization problems and some literature reviews have been published (Bishop, 1980; Presmeg, 2006). Dreyfus (1991) summarized the findings of some studies on specific difficulties in visualization, and their relationship with the intervening conceptual structure. He emphasized the importance of rules and conventions involved in the interpretation of different types of diagrams and figures that are not accessible to students. He also argued that the status of visualization should be recognized as a tool for learning. Arcavi (2003) examined the different roles that visualization can play in doing and learning mathematics, and how visual moderated sequences can be used to analyze procedures elicited by visual cues, he also pointed out that students may "see" in a representation something other than what an expert would, and related this to their conceptual structure, as suggested in Dreyfus (1991). Further, he related some difficulties in visualization to a lack of flexible translation between representations.

We agree with the above mentioned authors on the importance of the use of representations, both in visualization of mathematical objects and in conceptual understanding. We also consider that these issues play an important role not only in constructing the graph of a two variable function but in understanding the notion of function of two variables as well. In this study we focus on the analysis of some geometrical aspects related to functions of two variables that we consider essential in their understanding. Our research questions are: 
1) What are students' conceptions of fundamental planes (By fundamental planes we mean planes of the form $x=c, y=c, z=c$, where $c$ is a constant) and how are they used to construct the kinds of subsets of $\mathrm{R}^{3}$ (sections, contours, projections) that are needed in the graphical analysis of functions of two variables?

2) How are students' conception of graphs of functions of two variables related to their knowledge of $\mathrm{R}^{3}$ and its subsets?

\section{Theoretical Framework}

Two conceptual frameworks inform the theoretical basis used in this study. Firstly, APOS theory is used to model the development of the concept of two variable functions and, secondly, semiotic representation theory provides the conceptual tools to analyze flexibility in the use of different representations and its role in understanding the mathematical ideas under consideration. As APOS theory has proved to be useful in giving a detailed description of the construction of several mathematical concepts, it can prove useful in understanding how the concept of two variable functions is constructed. This theory does not include any specificity related to the role of representations in conceptual understanding, which we consider essential. In particular, the analysis of graphs of functions, together with other representations, is fundamental in building strong relationships among the components of a possible schema for the concept of two variable function. The description of treatments and conversions as actions or processes to perform with representations such as graphs or symbols adds specificity and a new angle of description to the analysis using APOS theory. These two conceptual frameworks can be used in a coherent and complementary way, both when analyzing students' responses and strategies 
when they work on tasks, and to design instruction in the future. We briefly describe both theories and give an example as a means to clarify the interaction between them.

In Action-Process-Object-Schema (APOS) theory (Asiala et al., 1996; Dubinsky, 1991, 1994), an action conception is a transformation of a mathematical object by individuals, according to an explicit algorithm which is conceived as externally driven. As individuals reflect on their actions, they can interiorize them into a process. Each step of a transformation may be described or reflected upon without actually performing it. An object conception is constructed when a person reflects on actions applied to a particular process and becomes aware of the process as a totality, or encapsulates it. A mathematical schema is considered as a collection of action, process and object conceptions, and other previously constructed schemas, which are synthesized to form mathematical structures utilized in problem situations (Baker, Cooley, \& Trigueros, 2000). These schemas evolve as new relations between new and previous action, process, and object conceptions and other schemas are constructed and reconstructed. Their evolution may be described by three stages that Piaget and García (1983) refer to as the "triad: At the general intra- stage some operational actions are possible, but there is an absence of relationships between properties. At the inter- stage, the identification of relations between different processes and objects, and transformations are starting to form, but they remain isolated. The trans- stage is defined in terms of the construction of a synthesis between them to form a structure (Cooley, Trigueros, \& Baker, 2007). Also, although it might be thought that in APOS theory there is a linear progression from action to process to object and then to having different actions, processes, and objects organized in schemas, this often appears more like a dialectical progression where there can be partial developments, passages and returns from one to other 
conception (Czarnocha, Dubinsky, Prabhu, \& Vidakovic, 1999). What the theory states is that the way a student works with diverse mathematical tasks related to the concept is different depending on his or her conception.

The application of APOS theory to describe particular constructions by students requires researchers to develop a genetic decomposition - a description of specific mental constructions one may make in understanding mathematical concepts and their relationships. Some clarifications are pertinent. A genetic decomposition for a concept is not unique, it is a general model about how a concept may be constructed, different researchers can develop diverse genetic decompositions of how students in general construct that particular concept, but, once one is proposed, it needs to be supported by research data from students. Frequently, gathered data reveal overlooked constructions which then give rise to a revised genetic decomposition.

As discussed before, visualization, representations and conceptual understanding can be considered as related among them and with the process of mathematics learning. The possibility of using concrete imagery needs to be coupled with rigorous analytical thought processes to be effective in mathematics learning (Dreyfus, 1991; Presmeg, 1997). Some authors consider that connections between internal constructs and representations where access is gained through the senses are involved in acts of visualization (Zazkis, Dubinsky and Dauterman, 1996), but visualization can be also considered in the case of connections among mental representations or images; in this case, mind-to-mind constructions can be thought of as described by interiorization, coordination, reversal, or encapsulation (Dubinsky, 1991). This position is supported by Duval $(1999,2006)$ who argued that thinking processes in mathematics require not 
only the use of representation systems, but also of their cognitive coordination. For him, reasoning and visualization are complementary thought processes. In Duval's analysis, understanding and learning mathematics require the comparison of similar semiotic representations. According to him, there are two different types of transformations of semiotic representations: treatments, which are transformations of representations that happen within the same representation register, and conversions which consist of changes of representation register without changing the object being denoted. He argues that these transformations are the source of many difficulties in learning mathematics, and that taking them into account helps in overcoming those difficulties: to compare similar representations and treatments within the same register in order to discriminate relevant values of the mathematical object so that the features that are mathematically relevant and cognitively significant are noticed, and to convert a representation from one register to another to dissociate the represented object and the content of the particular representation introduced so that the register does not remain compartmentalized.

The use of APOS and semiotic representation theory allows us to look at the same phenomena from two different but complementary perspectives. In APOS theory, treatments can be partially described in terms of actions or processes on an object within a specific representation register. Reflection on these actions may put forward aspects or properties of the object that are significant when those actions are interiorized in a process. Adding the point of view of semiotic representations theory enables the researcher to make the role of those actions in the construction of mathematical knowledge clear by emphasizing the need to discriminate all those features that are characteristic of the object in the register where the actions are being performed. Conversions may be described in APOS as relationships between different objects within a 
schema or as the interiorization into a process that makes it possible to consider the actions of comparison and identification between an object in two different representation registers as a mental association. Again, semiotic representation theory complements these considerations by pointing out its necessity in terms of cognitive importance. Also, both theories can be seen as coherent when one considers that a schema in APOS may contain the representations of a process or object in different registers, as has been stated above, while a treatment within a register or conversions within different registers in semiotic representation theory may involve objects in different schemas. Since in this study we are focusing on the geometric representation of functions of two variables, it is useful to be able to refer to the treatments and conversions between different representational registers without losing the genetic perspective afforded by APOS theory. An example may help to illustrate the relationship between both theoretical perspectives. When the action of substituting a number is performed on the function given by $z=$ $x^{2}+y^{2}$ to obtain $z=y^{2}$ or $z=1+y^{2}$, students may recognize these equations as quadratic functions in $\mathrm{R}^{2}$ which have been translated in the $\mathrm{y}-\mathrm{z}$ plane, and interiorize them into the process in which any number can be substituted to consider any translation of the function. Semiotic representation theory focuses on the consideration that this process involves a family of functions that have common characteristics. When considering the conversion of such equations into their graphs in the plane, the relationship between different objects, an equation and its graph, is constructed, and those aspects of the equation and the graph that remain invariant or change can be considered. In this case both objects, the analytical representation of the function and its graphical representation, are related within the same schema for functions of one variable. Consider now the mental transformations that might take place when considering the same actions on the function of two variables and their relationship with its graph in three 
dimensional- space. The above mentioned conversion of the equation into its graphical representation, obtained by the substitution of a specific number for the variable $x$ in the equation (for example, number 1), may need the consideration of the relationship between two different schema: $\mathrm{R}^{2}$ and $\mathrm{R}^{3}$ since it is necessary to consider the position of the quadratic function obtained on the fundamental plane $x=1$, when the actions of substitution of numbers and their conversion to the graphical register are interiorized, the resulting process involves the coordination of the vertical translations within the plane and the translation of the plane through the $\mathrm{x}$ axis. When this process is generalized all those curves can be considered as the intersection of the yet unknown surface $z=x^{2}+y^{2}$ with different planes and can be encapsulated into a graphical object that represents the surface, that is, the graph of the function of two variables.

When we chose in this study to restrict our attention to the geometrical aspects of functions of two variables, we are also choosing to keep track of issues related to representation and visualization that arise in the learning process, hence our choice of incorporating semiotic representation theory, while APOS theory allows us to examine the construction of the mathematical objects involved in the construction of functions of two variables and to describe the result of the coordination of different schemas.

The present study is part of an investigation of student understanding of the concept of function of two variables. As a first step in the study, the following preliminary genetic decomposition, based on theoretical consideration and the experience of authors as teachers, was proposed: - The schemas that we consider students should have developed previously, to be able to understand the concept of two variable functions, are: a) Intuitive three dimensional space which 
consists of a construction of the external material world; b) Cartesian plane which includes the concept of points as objects and relations between variables, such as curves, functions, and regions as processes resulting from the generalization of the action of representing their component points; c) Real numbers which includes the concept of number as an object, and arithmetic and algebraic transformations as processes; d) Sets ; e) Real functions of one real variable including function as process, operations with functions, and coordination of the analytic and geometric representation of functions.

- The Cartesian plane, real numbers, and the intuitive notion of space schemata must be coordinated in order to construct the Cartesian space of dimension three, $\mathrm{R}^{3}$, through the action of assigning a real number to a point in $\mathrm{R}^{2}$, and the actions of representing the resulting object both as a 3-tuple and as a point in space and making conversions between them. These actions are interiorized into a process that considers all the possible 3-tuples and subsets of 3-tuples, and their representation, to construct a process that when coordinated with the respective verbal, analytic, tabular and geometric representations can be thematized as three dimensional space, $\mathrm{R}^{3}$. - This space schema is coordinated with the schemas for function and set through the action of assigning one and only one specific height to each point in a given subset of $\mathrm{R}^{2}$, either analytically or graphically. This action is internalized into the process of assigning a height to each point on a subset of $\mathrm{R}^{2}$ to construct a two variable function, and the process of conversion needed to relate its different representations. When this process is generalized to consider any possible function of two variables, as a specific relation between subsets of $R^{2}$ and $R$ is encapsulated, the notion of two-variable functions has been constructed as an object. 
We suggest in the genetic decomposition that a schema for $\mathrm{R}^{3}$ and flexibility in the use of representations are necessary conditions to understand the concept of two variable functions, and specifically to be able to analyze graphs of such functions. We conjecture that fundamental planes mediate the process of conversion between analytical, geometrical and verbal representations of functions of two variables.

\section{Method}

APOS theory can be used in research in two different ways. One can use the theory to study students' constructions after they have finished a course or a series of courses related to the topic under study (Czarnocha, Dubinsky, Loch, Prabhu, \& Vidakovic, 2001; Dubinsky, Weller, Mc Donald, \& Brown, 2005; Trigueros, 2000). One can also use the theory to design activities to teach the topic and then analyze which constructions were made by students and which were not (Brown, De Vries, Dubinsky, \& Thomas, 1998; Dubinsky \& Yiparaki, 2000; McDonald, Mathews, \& Strobel , 2000). In both cases, results of the analysis can be used to refine the genetic decomposition so that it better reflects the way students, in general, construct those concepts. This study used the first approach with the intention of analyzing students' constructions related to the relationship of the $\mathrm{R}^{3}$ schema with the two variable function schema, after they had finished an undergraduate course on multivariate calculus for Applied Mathematics, Engineering or Economics majors at a private university. The teaching in that course was traditional. Some of these students had taken a course in Analytic Geometry in high school where they were introduced to the equations of the line and the conic sections in the plane, with an emphasis in algebraic manipulation. Nine students, three good, three average, and three weak students, were chosen by their instructor at the end of the semester to be interviewed, 
based on their grades and participation in the course. It is not assumed here that all the constructions developed by these students were developed during that course. They had already taken other courses that may have contributed to their constructions.

An instrument was designed to conduct semi-structured interviews. The selection of the tasks to be included in the interview was made on the basis of their possibility to inform on specific components of the genetic decomposition, and to test the conjecture of the mediation role played by fundamental planes, as well as students' capability to do treatments and conversions of representations in a flexible way. Some questions in the instrument were designed to be similar to those used in the course, others were different with the purpose to study what students could do in non familiar situations. The interviews were 45-60 minutes long; they were audio-taped and their transcription, and all the work produced by students were independently analyzed by the two researchers. Results were negotiated between them. The 12 questions of the interview were related to two variable functions. For the purpose of this study only 5 of those questions, related to subsets of $\mathrm{R}^{3}$, and graphs are discussed.

1. a. Represent, in three dimensional space, the point that is obtained when the ordered pair $(1,2)$ is assigned a height $z=3$.

b. Represent points $(0,-2,2)$ and $(-3,2,-2)$.

c. Find the coordinates $(x, y, z)$ of point $A$ represented in Figure 1:

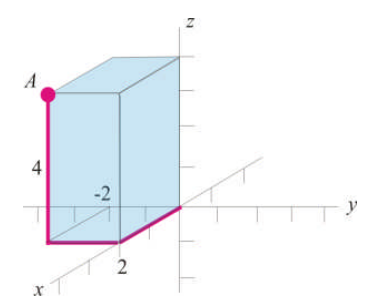


Figure 1

This question had the purpose of probing if students were able to perform the actions of assigning a height to a point in $\mathrm{R}^{2}(\mathrm{a})$, and representing given points in $\mathrm{R}^{3}(\mathrm{~b}, \mathrm{c})$, as well as their capability of converting between symbolic and graphical representation registers (c).

2. In this problem we refer to positive $x$ as east, negative $x$ as west, positive $y$ as north, negative $y$ as south, positive $z$ as up, negative $z$ as down.

a. Starting at point $(2,-1,4)$ move the point 4 units west, 3 north and 2 down. At what point does it end?

b. How would you move point $(-3,4,-1)$ to end at point $(2,-2,2)$ ?

c. If you start at point $(3,-2,-4)$ and move freely in the directions east-west and up-down, give an equation that describes the set of all points that may be reached.

This problem intended to determine if students could perform the actions or process needed to translate a point in $\mathrm{R}^{3}$; if they could recognize treatments on a point in $\mathrm{R}^{3}(\mathrm{a}, \mathrm{b})$ and if they were able to generalize the results of their actions or process into geometrical object in $\mathrm{R}^{3}$ (a plane); visualize the result of a treatment and perform a conversion (c).

3. a. Draw the set of points in $\mathrm{R}^{2}$ that satisfies the equation $x^{2}+y^{2}=1$.

b. Draw in $\mathrm{R}^{3}$ the set of points that is the result of assigning a height $z=3$ to the points in $\mathrm{R}^{2}$ satisfying equation $x^{2}+y^{2}=1$.

c. Draw in $\mathrm{R}^{3}$ the set of points that may be reached by starting with points in $\mathrm{R}^{2}$ that satisfy equation $x^{2}+y^{2}=1$ and assigning them an arbitrary height $z$.

The goal of this question was to verify if students could coordinate their schemas for Cartesian plane, real number and their intuitive notion of three dimensional space, through actions $(\mathrm{a}, \mathrm{b})$ or 
a process (c), conversions from a symbolic representation to a geometric one (a), and to visualize the result of a treatments $(b, c)$.

4. Consider the surface given in Figure 2:

a. Draw on a two-dimensional plane the points on the surface that satisfy $z=1$.

b. Draw on a two-dimensional plane the points on the surface that satisfy $x=1$.

c. Draw on a two-dimensional plane the points on the surface that satisfy $y=1$.

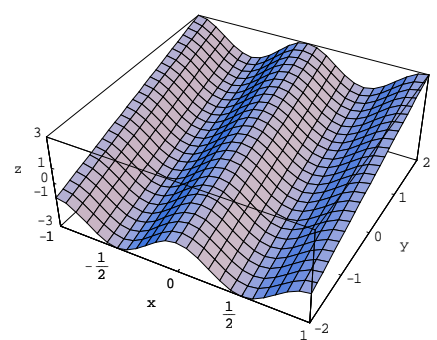

Figure 2

The response to this problem requires the coordination of subsets of $\mathrm{R}^{3}$ and a reversal process of coordination of subsets of $\mathrm{R}^{3}$ with the Cartesian plane $\mathrm{R}^{2}$. This is a difficult question, it involves visualizing the intersection between surfaces, which is a treatment in the geometrical register of representation and graphically representing the result of that intersection, which involves another treatment. Constructions such as the one this problem asks for are needed when analyzing graphs of two variable functions.

5. In each of the following problems, represent the given set in three-dimensional space as carefully as you can. Also describe it verbally giving as much detail as possible.
a1. $\{(x, y, 0): x=3\}$
a2. $\{(x, y, z): x=3\}$
b1. $\left\{(x, y, z): z=x^{2}+y^{2}, y=3\right\}$
b2. $\left\{(x, y, z): z=x^{2}+y^{2}, z=4\right\}$ 
This question involves the coordination of subsets of $\mathrm{R}^{3}$ with their graphical representation and descriptions in natural language which are conversions among different representation registers. It intended to verify students' capability of making sense of set notation, and if they could coordinate the set and $\mathrm{R}^{3}$ schemata.

\section{Results}

Interviewed students showed differences in their construction of the schema of functions of two variables when their responses to the whole instrument are considered. Results show that all of the students demonstrated they had constructed a schema for $\mathrm{R}^{3}$ which includes points as objects in their different representations. Differences in their capability to graph two variable functions and to interpret those graphs can be related to differences in structure of their $\mathrm{R}^{3}$ schema, that is, in the relationships they have been able to construct between elements of this schema; this in turn is intimately related to their capability to do treatments and conversions among different representation registers, and to foresee and visualize the result of actions on particular representation registers. The nine students interviewed fell into three groups, each with three students, who demonstrated difficulties with different aspects of the genetic decomposition, but similarities in the structure of the $\mathrm{R}^{3}$-schema revealed by their responses. This grouping differed from that proposed by their teacher: during the interview, one "weak" student demonstrated a better understanding and one "good" student demonstrated less understanding. Results of the analysis showed that the three resulting groups could be related with different levels of the Cartesian space schema. In the description that follows we present the analysis of results according to such levels. 


\subsection{Students who demonstrated an Intra-level structure of the Cartesian space schema}

The students in this group showed many difficulties with the graphical representation of two variable functions, and could not coordinate their schema for an intuitive notion of three dimensional space and that for $\mathrm{R}^{3}$, as suggested by the genetic decomposition. Rodrigo exemplifies typical responses of this group of three students. When working with problem $2 \mathrm{c}$, and after taking a lot of time to think, Rodrigo responded:

Rodrigo- (reads the problem) ... ah, ok, ... that is, we can only move freely on the x axis... and on the $z$ axis but the $y$ axis is fixed, ok ... then it would be ... [long pause]

Interviewer- what are you thinking?

Rodrigo - I'm thinking that we are fixed at y, aren't we? It does have point 3, say here, and I have to describe an equation so that I may move freely on $x$ and on $z$, so that if we are in three dimensions, it would take ... here ... we form a plane or not, there it is ... negative ...ok Interviewer - ok, so you wrote $y=x+z+3$

Rodrigo-3, what happens is that, according to me, if I write $y=x+z+3$, then it works here that, that it is $x$ at $3 \ldots$

This response demonstrates that he was neither able to do a treatment to the point in the graphical register, nor to describe the result of the needed actions. He went back to a memorized fact, the equation of a plane, and could not relate it to the task. He was not able to relate the set of points generated by the movement of the given point in space to the equation of the corresponding fundamental plane. Later, the interviewer asked Rodrigo to represent the plane $y=$ -2 in a physical model of three dimensional space but he was unable to do it and to relate it to the expected solution of the question. In several instances he recognized equations of the form $x=c$, $y=c$, and $z=c$ as planes in $\mathrm{R}^{3}$, but could not perform treatments on those planes when the tasks 
required it or convert these memorized equations to their geometric representation. $\mathrm{He}$ demonstrated he had an action conception of planes and difficulty coordinating his schema of $\mathrm{R}^{3}$ with his schema of intuitive three dimensional space.

All students in this group had similar problems as Rodrigo. They used disconnected memorized facts in their responses, and didn't show evidence of having constructed relations between their actions and their intuitive knowledge of space. They struggled when asked to do treatments and conversions, independently of the representation register used. Their difficulties visualizing the results of their actions seem to be related to lack of generalization of their actions on points in space into a process that considers all the possible 3-tuples that satisfy a specific restriction. The $\mathrm{R}^{3}$ schema they seem to have constructed includes points and their representations, some memorized facts about fundamental planes, and other planes, but the fact that they cannot do any treatments and conversions on sets of points reveals that they have not constructed relationships between these components of the schema (Intra-level structure of schema).

\subsection{Students who demonstrated an Inter-level structure of the Cartesian space schema}

Students in this group were able to consider some sets of points in $\mathrm{R}^{3}$, such as fundamental planes, projections and some surfaces, but showed difficulties when they needed to coordinate them. For example, referring to question 2c, Maria deduced the equation of the plane very quickly. She responded:

Maria- ok and since I don't have north and south then ... the plane $y=-2$.

Interviewer-would that be the equation?

María- ... y = -2 
Consistently throughout the interview she was able to visualize fundamental planes and to make conversions from their analytic representation to the corresponding geometric representations. However in problem 5 part b1:

Maria- we have that $z=x^{2}+y^{2}$ with $y=3 \ldots z=x^{2}+y^{2}$ is like, like a cup, and $y=3 \ldots$ here is $y$ $=3$, then it is all 3-tuples of $x, y, z \ldots$

Interviewer- how would that look?

Maria- ... something like this, that is the ... that is, y can only take 3 as a value? ... then it would be ... a paraboloid ... over this, over 3 and then here they are 3.

Interviewer- What is the relationship between this set and the cup you mentioned earlier?

Maria-Well, it is like the tip, isn't it? that is the ... the ... but no, because ... yes because $x$ can be positive or negative, but in ... that is, it can have any $x$ or any $y$, any $x$ but in $y$ it can only be 3, like a piece ... a slice of the piece

Interviewer- here you drew a circle, does it include the points inside the circle?

Maria- ... well in $x$ it has ... it has no point because there is no such ... y and $z$, well neither.

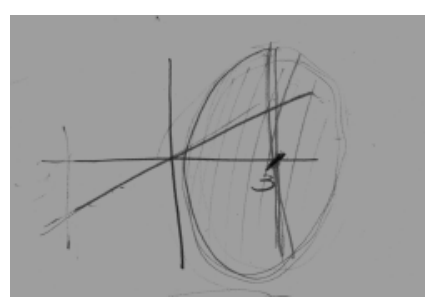

Figure 3: Maria's attempt at drawing $\left\{(x, y, z): z=x^{2}+y^{2}, y=3\right\}$

Maria had difficulty representing the $\operatorname{set}\left\{(x, y, z): z=x^{2}+y^{2}, y=3\right\}$ graphically, although she was able to recognize $y=3$ as a plane. This can be seen in her drawing of what looks like a disc inside the plane $y=3$ (see Figure 3). Her inconclusive answer to the last question together with the solid circle she drew confirms that even though she was able to do the conversion needed 
from the analytic to the geometrical register of both surfaces, the paraboloid and the fundamental plane, independently, she was not able to coordinate both surfaces to find their intersection, nor to visualize its result, the transversal section. This lack of coordination made it impossible to her to represent the intersection geometrically. The use of the word "slice" while analyzing transversal sections of surfaces, seemingly made her relate her actions to her schema for intuitive three dimensional space, and associate an image of a 3D solid "cup" with the expression $z=x^{2}+y^{2}$ thus obtaining a surface as the intersection, instead of a curve resulting from the coordination involved in intersecting both surfaces.

Later, in order to describe the set of points in $\mathrm{R}^{2}$ that satisfy the equation $x^{2}+y^{2}=1$ when assigned a height of 3 , she said:

Maria- ...ah then ... we'd be left with ... something like this ... in the interior [see Figure 4, she drew a cup-like surface that she quickly fixed to have her drawing look like a cylinder] Interviewer- then, that looks like a surface?

Maria-yes, this is a surface because we are in $\mathrm{R}^{3} \ldots$ it is ... there... a cylinder...

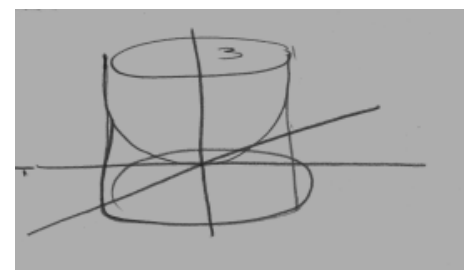

Figure 4: Maria's points on $\mathrm{R}^{2}$ that satisfy the equation $x^{2}+y^{2}=1$ and are assigned a height of 3 While working with question 3, Maria showed difficulties converting from a description in natural language to the geometrical register. This difficulty can also be related to difficulties in predicting and visualizing the result of actions on curves which are related to the coordination of 
a conversion and a treatment when both of them are needed. She was not able to determine that the result of lifting a curve is also a curve.

When working with the graph given in problem 4 , she initially struggled with the section corresponding to $x=1$, but eventually succeeded with some help, however, when working with the section corresponding to $y=1$ :

Interviewer- and how will that look?

Maria- ... well ... from ... so $x$ from -1 to 1 ... and it didn't touch any from 0 to 1 in $x$ and in $y . .$. now I'm lost, I'm lost

Interviewer- and how is that? a surface? a curve? ...

Maria- no, a curve

Interviewer- a curve, could you draw that curve, more or less?

Maria- ... from -1 to 1 in $x$... and at the points where y has the value 1 ...[long pause] ... I don't know, it would look, like a curve ... that is, they only go through 1 in y and from -1 to 1 in $x$ ... would be these ones here

Her final drawing is shown in Figure 5.

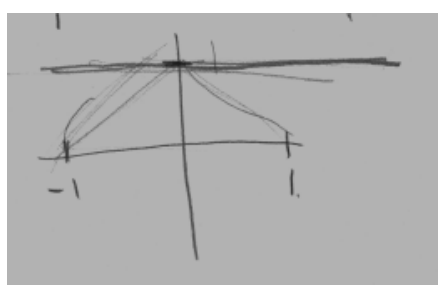

Figure 5: Maria's drawing of the points on the surface of problem 4 that satisfy $y=1$.

Maria showed difficulty representing geometrically contours and transversal sections of surfaces, which involve coordination of treatments in the graphical register and conversion between 
representation registers. She was unable to represent the section corresponding to $y=1$ in problem 4 , even though she recognized $y=1$ as a plane, and then the section corresponding to $y=3$ in question 5, again, she recognized both surfaces but was unable to coordinate them to find their intersection, although, with a more familiar problem, in the case of problem $5 \mathrm{~b} 2$, she was able to do this coordination correctly.

Results show that students in this group had coordinated their $\mathrm{R}^{3}$ schema with those for sets and one variable functions, they had interiorized the processes needed to identify some subsets of $\mathrm{R}^{3}$ such as lines, fundamental planes and some surfaces. They also had constructed relationships between components of the Cartesian space schema which were demonstrated by the interiorization of actions on points to consider curves or surfaces, and also by coordinations by means of treatments and conversions among different representation registers. However, as shown with difficulties Maria had, they struggled when coordination between treatments or between treatments and conversions were involved or when they needed to coordinate fundamental planes with the schema of subsets of $\mathrm{R}^{3}$, mainly surfaces, and that of the Cartesian 2D plane to produce projections and intersections of surfaces. This coordination through treatments and conversions is important when it is necessary to visualize the result of specific actions on equations or graphs, and to analyze or draw graphs of functions of two variables.

\subsection{Students who demonstrated being in transition to the Trans-level structure of the Cartesian} space schema

The three students who demonstrated a good understanding of graphs of two variable functions, showed that they had constructed all the coordinations described in the genetic decomposition 
required for the construction of $\mathrm{R}^{3}$ and its subsets; also that their schema for $\mathrm{R}^{3}$ consisted of points, planes, and surfaces as interrelated objects. When working in problem 4, Rafael, for example, commented:

Rafael-let's see, draw on a plane of two dimensions the points on the surface that satisfy $x=1$, $x=1$ would be $\ldots$ here we have $x=1 \ldots x=1$ would simply be this side [correctly pointing to one side of the boxed surface] $\ldots x=1$ would simply be all this line ... all this line that is on the $x y$ plane ... $x y$... in $x=1 \ldots$ now ... the convenient plane is ... the plane would be $x, x z$ and it would have a line that goes from here ...

The line he drew is close to being correct, but on an $x z$ plane. Again on part $\mathrm{c}$, he drew the curve correctly but mislabeled the plane as $y z$ (see Figures 9 and 10).

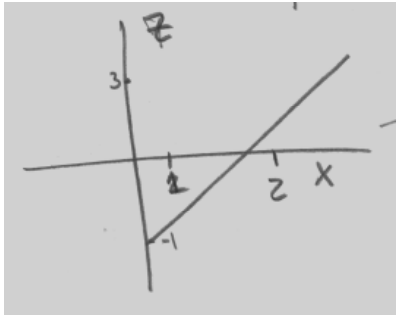

Figure 9

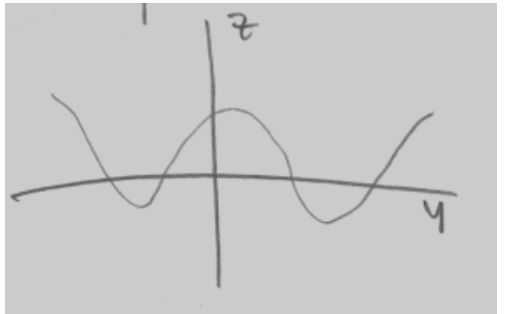

Figure 10

When he was trying to draw the contour corresponding to $z=1$, he said:

Rafael-... I first locate the axes, the x axis, the y axis, this is y zero, ahh now now now now, this $y$ zero, this $x$ zero ... these, the axes and $z \ldots z$, ok, consider in a two dimensional plane ... points on that satisfy $z=1 \ldots$ let's see, in a two dimensional plane, the points on the surface that satisfy $z=1, z=1, \ldots z=1 \ldots$ I am at $z$ minus one, plus one, let's see, here I would try to draw a plane parallel to $x y$, but finding the points of intersection would be somewhat complicated, if I didn't have the equation of this, of this surface ...

Rafael clearly described the process of intersection between the surfaces, he referred to a plane "parallel to the $x y$ plane" and to "finding the points of intersection", but he was not able to 
visualize and graph the result of this intersection. He only struggled when representing graphically the harder intersection, even though he was able to describe the process verbally. This showed that coordination of fundamental planes and surfaces of $\mathrm{R}^{3}$ through the process of intersection of surfaces was on the way of being encapsulated.

The three students in this group showed that their schema included some coordination between successive conversions and treatments as well, as relationships between different components of $\mathrm{R}^{3}$ demonstrating they were in transition to Trans-level of the schema.

\section{Discussion}

Results of this study show that most of the interviewed students had constructed a schema for $\mathrm{R}^{3}$, a construction that we hypothesized as fundamental in the genetic decomposition of the concept of two variable functions, even though they had some problems with the sub-schema of subsets of $\mathrm{R}^{3}$. Students in the first group described had difficulties in understanding fundamental planes and other subsets of $\mathrm{R}^{3}$. They had not interiorized actions consisting of memorized facts, into the process necessary to understand those subsets, and they could not relate them to sets of points in space. They did not show any evidence of coordination of the constructions necessary to be able to flexibly relate different representations of objects in space and to perform actions on any object, other than points, in $\mathrm{R}^{3}$. A source of difficulty for these students seems to be a lack of coordination between their schema of $\mathrm{R}^{3}$ and schemas for one variable functions and sets. This makes it difficult for them to do treatments on curves or surfaces in any representation register, not to say conversions between registers. 
Among those students who were able to perform actions on sets of points in $\mathrm{R}^{3}$, as students in group two, results showed that visualizing and graphing the result of those actions was more difficult than is sometimes acknowledged by instructors. It seems that students' intuitive notion of space interferes when students try to predict the result of a treatment or a conversion of an object in $\mathrm{R}^{3}$, independently of the representation register used. This interference seems to be related to the use of language. It was shown that sometimes the use of certain words such as "cutting a surface" or "lifting a curve" made students visualize and represent an object related to their common experience rather that the mathematical object expected. It seems to be important that the meaning of this kind of sentences be explicitly discussed in class. It was also observed that the possibility of visualizing actions on curves and surfaces, and their result, as in the use of fundamental planes to construct sections, contours and projections, seems to require an object conception of fundamental planes and curves, together with the ability to flexibly make treatments within the analytical and graphical representations of, at least, simple objects.

Even the students who showed a better understanding of graphs of two variable functions demonstrated difficulties when they had to visualize, describe or represent the intersection of a fundamental plane with a given surface. Most students struggled when both the surface and the plane were given algebraically, and when the plane was given algebraically and the surface geometrically. This difficulty was not predicted in the genetic decomposition described above and should be considered in its future refinement. Only one student could flexibly intersect a fundamental plane with a surface, and use different representations related to that intersection. This ability is important to analyze graphs and to interpret some relevant results regarding these functions, such as limits and derivatives, but seems not to have been developed by these students 
during their course. This ability requires, according to our analysis, the possibility to do or imagine doing a succession of treatments and conversions of representations of the function of interest. This also seems to have an effect in building a more coherent structure of a mathematical conception of $\mathrm{R}^{3}$ and of graphs of two variable functions. We consider that instructors must pay more attention to be sure that the abilities to relate treatments between representations and to convert between different representations, including tables, that were not analyzed in this part of the study, but are no less important, are developed by students before using them to explain other abstract concepts related to these functions. We agree with Gagatsis, Christou, and Elia (2004) and with Dreyfus (1991) who considered that "Diagrams and figures contain relevant mathematical information in a form that is determined by certain rules and conventions ... They are therefore not accessible to students who have not had the opportunity to get acquainted with these rules and conventions" (p. 34).

Visualization and a flexible use of different representations seem also to be related to reasoning by analogy. Some of the interviewed students, as Maria and students from the third group, used it, however, it seems that this is an ability that has to be taken more explicitly into account by instructors in order for students' to develop a deeper understanding of these functions. Also, representations of $\mathrm{R}^{3}$ always require the ability to interpret and draw projections in $\mathrm{R}^{2}$. Students' difficulties are evidence that this capability is difficult to acquire without explicit experience on it. Some of the interviewed students were helped during the interview by the use of concrete materials they could manipulate. We consider the use of such materials should also be considered in instruction, as suggested by Bishop (1983). 
Analysis of the results of this study shows that the flexible use of different semiotic representation registers is related, as stated by several authors, to the cognitive development of students. Furthermore, it shows how the use of semiotic representations theory can put forward some important relationships that need to be taken into account when describing the constructions described by the genetic decomposition of the notion of two variable functions based on APOS theory. These two theories direct their attention to different aspects of mathematics learning process that, in the case of the study of graphs of two variable functions, are intimately related. The complementary use of these two theories was not only useful but fruitful in this analysis.

\section{Conclusions}

This study gives information about students' difficulties and understanding of functions of two variables. In particular, results obtained show that the generalization of understanding of one variable functions to two variable functions, in particular in the case of graphical representation, is not direct.

This study gives evidence that the understanding of graphs of functions of two variables is not easy for students and that it can be related to the structure of students' schema for $\mathrm{R}^{3}$, specifically with the construction of subsets of points in space. The construction of relationships between components of students' schema has been found to be needed in order to be able to flexibly relate different representation registers and construct coordinations, which are needed to understand the graphical representation of functions of two variables. 
It was found that the possibility of intersecting surfaces with planes, and predicting the result of this intersection plays a fundamental role in understanding graphs of two variable functions and was particularly difficult for students. The fact that this relation was only constructed by one of the interviewed students seems to indicate that a more consistent effort is needed when teaching this subject in multivariate calculus courses.

From the theoretical point of view, the use of semiotic representation theory and APOS theory was useful and complementary. On the one hand, analyzing the way students worked within representations, provided information about the kind of relationships among components of students' schema that had been constructed, and about mind to mind visualization which would have been difficult to achieve using only APOS theory. On the other hand, APOS constructs were used to specify the necessary constructions for students to be able to work within and among representations. We expect that the results obtained in this study can be useful in designing activities to teach functions of two variables, and that their use will contribute to a more significant learning of this concept.

\section{Acknowledgements}

This project was partially supported by Asociación Mexicana de Cultura AC We are thankful to Ed Dubinsky for his comments and suggestions.

\section{References}

Arcavi, A. (2003). The role of visual representations in the learning of mathematics. Educational Studies in Mathematics, 52(3), 215-241. 
Asiala, M., Brown, A., DeVries, D. J., Dubinsky, E., Mathews, D., \& Thomas, K. (1996). A framework for research and development in undergraduate mathematics education. In J. Kaput, E. Dubinsky, \& A. H. Schoenfeld (Eds.), Research in collegiate mathematics education II (pp. 1-32). Providence, RI: American Mathematical Society.

Baker, B., Cooley, L., \& Trigueros, M. (2000). The schema triad - a calculus example. Journal for Research in Mathematics Education, 31, 557-578.

Bishop, A. J. (1980). Spatial abilities and mathematics education: A review. Educational Studies in Mathematics, 11(3), 257-269.

Bishop, A. J. (1983). Space and geometry. In R. Lesh, \& M. Landau (Eds.), Acquisition of Mathematics Concepts and Processes (pp. 175-203). New York: Academic Press.

Breidenbach, D., Hawks, J., Nichols, D., \& Dubinsky, E. (1992). Development of the process conception of function. Educational Studies in Mathematics, 23, 247-285.

Brown, A., DeVries, D., Dubinsky, E., \&Thomas, K. (1998). Learning binary operations, groups, and subgroups. Journal of Mathematical Behavior, 16(3), 187-239.

Cooley, L., Trigueros, M., \& Baker, B. (2007). Schema thematization: A framework and an example. Journal for Research in Mathematics Education, 38, 370-392.

Czarnocha, B., Dubinsky, E., Prabhu, V., \& Vidakovic, D. (1999). One theoretical perspective in undergraduate mathematics education research. In O. Zaslavsky (Ed.), Proceedings of the 23rd Conference of PME (Vol. 1, pp. 95-110). Haifa, Israel: PME.

Czarnocha, B., Dubinsky, E., Loch, S., Prabhu, V., \& Vidakovic, D. (2001). Conceptions of area: In students and in history. The College Mathematics Journal, 32(2), 99-109.

Dreyfus, T. (1991). On the status of visual reasoning in mathematics and mathematics education. In F. Furinghetti (Ed.), Proceedings of the 15th Conference of the International Group 
for the Psychology of Mathematics Education (Vol 1, pp. 33-48). Genova: Universita de Genova.

Dubinsky, E. (1991). Reflective abstraction in advanced mathematical thinking. In D. Tall (Ed.), Advanced mathematical thinking (pp. 95-123). Dordrecht: Kluwer Academic Press.

Dubinsky, E. (1994). A theory and practice of learning college mathematics. In A. Schoenfeld (Ed.), Mathematical thinking and problem solving (pp.221-243). Hillsdale, NJ: Erlbaum.

Dubinsky, E., Weller, K., McDonald, M. A., \& Brown, A. (2005). Some historical issues and paradoxes regarding the concept of infinity: An APOS analysis, Part 1. Educational Studies in Mathematics, 58(3), 335-359.

Dubinsky, E., \& Yiparaki, O. (2000). On student understanding of AE and EA quantification. In E. Dubinsky, A. H. Schoenfeld, \& J. Kaput (Eds.), Research in collegiate mathematics education IV (pp. 239-289). Providence, RI: American Mathematical Society.

Duval, R. (1999). Representation, vision and visualization: Cognitive functions in mathematical thinking. In F. Hitt, \& M. Santos (Eds.), Proceedings of the XXI Annual Meeting of the North American Chapter of the International Group for the Psychology of Mathematics Education (pp. 3 - 26). Columbus, OH: ERIC.

Duval, R. (2006). A cognitive analysis of problems of comprehension in a learning of mathematics. Educational Studies in Mathematics, 61(1), 103-131.

Gagatsis, A., Christou, C., \& Elia, I. (2004). The nature of multiple representations in developing mathematical relationships. Quaderni di Ricerca in Didattica, 14, 150-159.

Goldin, G. A. (1998). Representational systems, learning, and problem solving in mathematics. Journal of Mathematical Behavior, 17, 137-165. 
Goldin, G. A. (2002). Representation in mathematical learning and problem solving. In L. D. English (Ed.), Handbook of international research in mathematics education (pp. 197218). London: Lawrence Erlbaum.

Gutiérrez, A. (1996). Visualization in 3-dimensional geometry: In search of a framework. In L. Puig, \& A. Gutiérrez (Eds.), Proceedings of the $20^{\text {th }}$ Conference of the International Group for the Psychology of Mathematics Education (Vol. 1, pp. 3-19). Valencia: Universidad de Valencia.

Hitt, F. (Ed.). (2002). Representations and mathematics visualization. North American Chapter of the International Group for the Psychology of Mathematics Education. Mexico City: Cinvestav-IPN.

McDonald, M. A., Mathews, D. M., \& Strobel, K. H. (2000). Understanding sequences: A tale of two objects. In E. Dubinsky, A. Schoenfeld, \& J. Kaput (Eds.), Research in collegiate mathematics education IV (pp. 77-102). Providence, RI: AMS.

Piaget, J., \& García, R. (1983). Psicogénesis e historia de la ciencia. Mexico: Siglo XXI Editores.

Presmeg, N. C. (1997). Generalization using imagery in mathematics. In L. D. English (Ed.), Mathematical reasoning: Analogies, metaphors and images (pp.299-312). Mahwah, New Jersey: Lawrence Erlbaum Associates.

Presmeg N. C. (2006). Research on visualization in learning and teaching mathematics. In A. Gutiérrez, \& P. Boero (Eds.), Handbook of research on the psychology of mathematics education: Past present and future (pp. 205-235). Rotterdam: Sense Publishers. 
Sfard, A. (1992). Operational origins of mathematical objects and the quandary of reificationThe case of function. In E. Dubinsky, \& G. Harel (Eds.). The concept of function: Aspects of epistemology and pedagogy (pp. 59-84). United States: MAA.

Trigueros, M. (2000). Students' conception of solution curves and equilibrium in systems of differential equations. In Fernandez, M. L.(Ed.), Proceedings of the XXII Annual Meeting of the North American Chapter of the International Group for the Psychology of Mathematics Education (pp. 93-97). Columbus, OH: ERIC.

Van Nes, F., \& De Lange, J. (2007). Mathematics education and neurosciences: Relating spatial structures to the development of spatial sense and number sense. The Montana Mathematics Enthusiast, 4(2), 210-229.

Yerushalmy, M. (1997). Designing representations: Reasoning about functions of two variables. Journal for Research in Mathematics Education, 28, 431-466.

Zazkis, R., Dubinsky, E., \& Dauterman, E. (1996). Using visual and analytic strategies: A study of students' understanding of permutation and symmetry groups. Journal for Research in Mathematics Education, 27, 435-457. 